\title{
Hematoma epidural lumbar crónico espontáneo
}

\author{
J.M. Belinchón, J. Campos, J. Merino, J.M. Gallego y C. Barcia
}

Servicio de Neurocirugía. Consorcio Hospital General Universitario, Universidad de Valencia

\section{Resumen}

Presentamos un caso excepcional de estenosis de canal lumbar con síndrome de cola de caballo por un hematoma crónico espontáneo en el espacio epidural L2-L3.

Se trata de un varón de 53 años que, sin ninguna clase de antecedente patológico relacionado con el problema actual (traumatismos, punciones lumbares, toma de antiagregantes o anticoagulantes), presenta una colección hemática epidural que va comprimiendo progresivamente la cola de caballo. Las pruebas de imagen mostraron una colección polilobulada, bien delimitada, que se interpretó como quiste sinovial. Tras la cirugía (laminectomía descompresiva y evacuación) se confirmó el diagnóstico con estudio histológico. Tras la operación se recuperó por completo la función neurológica.

PALABRAS CLAVE: Hematoma epidural. Crónico. Lumbar. Espontáneo. Laminectomía.

\section{Chronic spontaneous lumbar epidural hematoma}

\section{Summary}

We report an exceptional case of cauda equina syndrome, caused by a chronic epidural hematoma of the lumbar spine, (L2-L3). A 53 year old man without history of trauma, lumbar punctures or antiagregant medication, suffered from progressive back pain and minor motor deficit in the legs. The etiology, MRI, intraoperative findings and microscopical study are presented and discussed. After surgery there was a complete resolution of the clinical picture.

KEY WORDS: Chronic. Epidural. Spontaneous. Hematoma. Laminectomy.

Recibido: 25-10-04. Aceptado: 17-03-05

\section{Caso clínico}

Varón, de 53 años de edad, que comenzó con dolor e impotencia en ambos miembros inferiores, adormecimiento de piernas con parestesias y en las últimas semanas dificultad para la marcha. Los síntomas se aliviaban parcialmente al acostarse y no empeoraban sentado. La sintomatología predominante era sensitiva, con intenso dolor en miembros inferiores.

El dolor aumentaba con maniobras de Valsalva y sufría empeoramientos cíclicos.

Todo el cuadro comenzó en Septiembre del 2002, un año antes de ser visto en consulta de Neurocirugía.

El paciente refería, como únicos antecedentes, dolores de espalda y una intervención de hernia inguinal bilateral hacía años, bajo anestesia general.

En las imágenes de Resonancia Magnética se observó, una colección polilobulada, intrarraquídea, extradural que comprimía el saco dural en el $90 \%$ de su volumen. Se informó por radiólogo como quiste sinovial L2-L3 (Figuras 1-2).

No se efectuó angiografía espinal, aunque ni en las imágenes de Resonancia ni en el acto quirúrgico se apreció ningún tipo de malformación vascular.

\section{Intervención}

Se efectúa una laminectomía desde L4 hasta L2. En L3 y L2 comienza a obtenerse fragmentos de tejido óseo y cartilaginoso, infiltrados por un líquido oscuro que, además, se derrama al campo operatorio con un aspecto similar al "fuel-oil". Se elimina el tejido de la zona, descomprimiendo el saco dural, y se procede al cierre de la incisión.

\section{Anatomía patológica}

El informe de Anatomía Patológica describe las muestras tanto extemporáneas como postoperatorias como tejido osteocartilaginoso, músculo y tejido fibroso, con zonas de hemorragia y macrófagos en la periferia cargados de pig- 


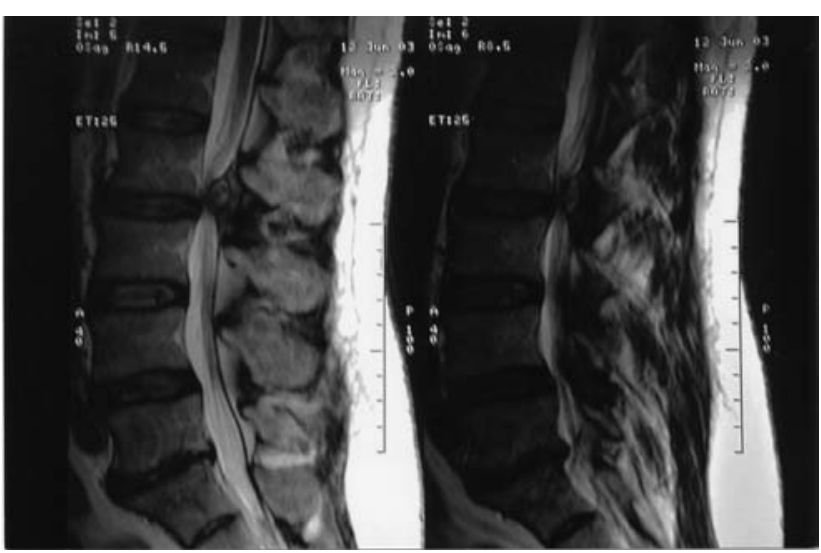

Figura 1. Imagen en T2,cortes sagitales. Imagen hipointensa en espacio epidural L2-L3 que comprime cola de caballo.

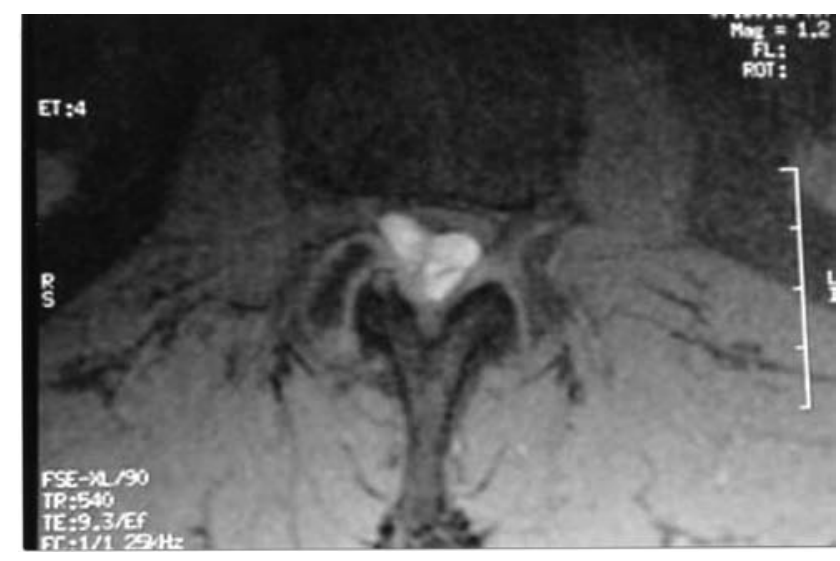

Figura 2. Cortes axiales mostrando la lesión polilobulada invadiendo canal medular. Imagen T2.

mento hemosidérico (Figura 4).

No se observan otro tipo de lesiones ni célula maligna o atípica alguna.

\section{Resultado postoperatorio}

El postoperatorio cursó con una recuperación excelente sin complicaciones y una mejoría total de los síntomas (Figura 3).

Tras un año de seguimiento el paciente permanece totalmente asintomático.

\section{Discusión}

El hematoma epidural espinal espontáneo supone una rara entidad nosológica ${ }^{12} \mathrm{y}$, dentro de esta patología, la cronicidad del proceso lo hace aún mas excepcional.

La etiología del hematoma epidural raquídeo puede ser diversa, pero en su mayoría (un tercio de los casos) se relacionan con los anticoagulantes orales ${ }^{3}$. También

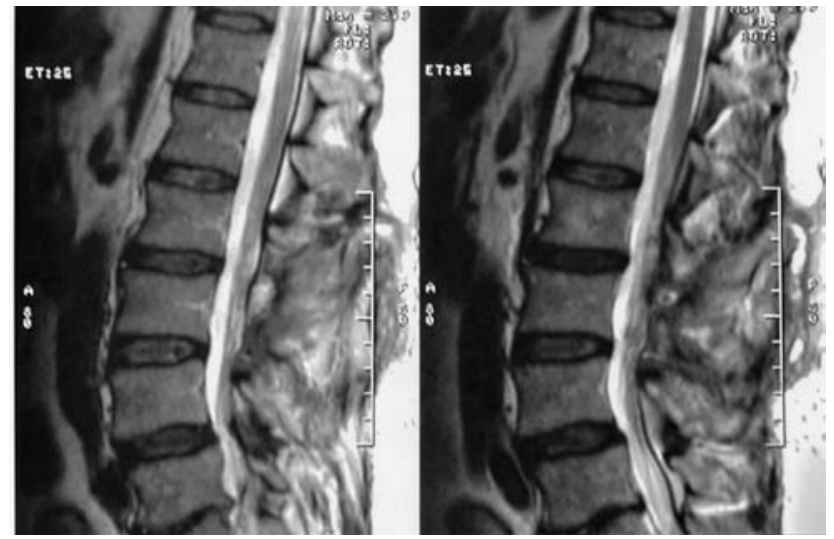

Figura 3. Resultado postoperatorio, laminectomía L2-L4. Descompresión canal medular.

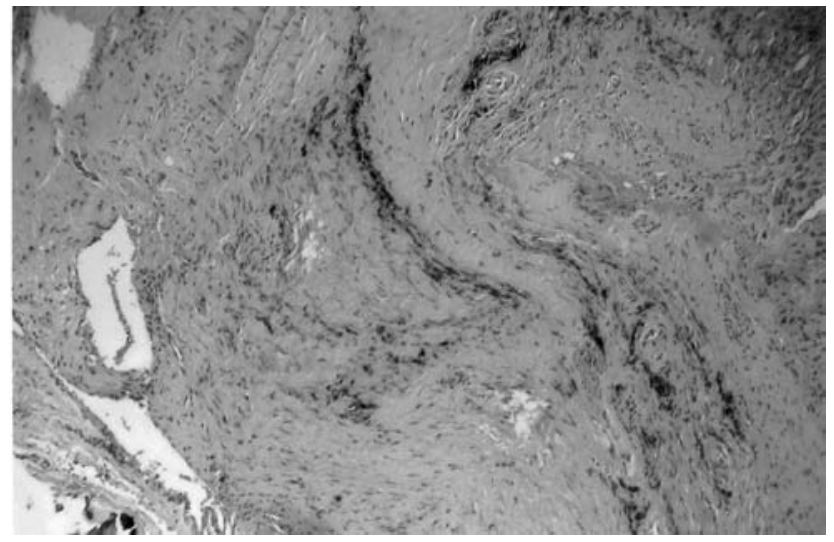

Figura 4. Tejido fibroso, depósitos de fibrina y hemosiderina.

se han implicado a los AINES ${ }^{15}$. Las formas traumáticas incluyen los casos producidos tras punción lumbar o anestesia epidural, casos prácticamente exclusivos de dichas maniobras, en pacientes que toman antiagregantes, están anticoagulados o padecen diátesis hemorrágicas ${ }^{2,11,14,16}$. Los casos espontáneos suelen relacionarse con malformaciones arteriovenosas o hemangiomas vertebrales ${ }^{4}$. Se han descrito algunas formas tras importantes esfuerzos físicos ${ }^{5,6}$, lesiones traumatológicas como espondilolisis ${ }^{9}$ o hernias discales ${ }^{1}$.

Los niveles lesionales más habituales son en columna dorsal, pero pueden producirse a cualquier altura del raquis. Con gran frecuencia se localizan en la zona más posterior del canal raquídeo, lo cual hace más fácil su evacuación quirúrgica ${ }^{3}$.

El cuadro clínico es consistente, pero poco específico; comienza habitualmente como dolor intenso de espalda con componente radicular, similar a una hernia discal. Según la naturaleza del proceso se desarrollará sintomatología de un modo brusco o, como en este caso, a lo largo de meses, empeorando progresivamente la clínica radicular. Los 
signos de déficit motor pueden quedar disimulados por el dolor.

Es muy rara la regresión espontánea, salvo en hematomas laminares de muy pequeño tamaño15, por lo que el tratamiento de elección es la laminectomía descompresiva ${ }^{3}$.

En los pacientes de alto riesgo quirúrgico (ejp. Anticoagulados) la morbilidad y la mortalidad son muy elevadas. Si se decidiese no operar deberá suspenderse la toma del anticoagulante y revertir el efecto si fuese necesario.

El caso presentado implica una entidad excepcional dentro de este tipo de patología, ya que no existía ningún tipo de agente o causa de las descritas que pudiera relacionarse con la aparición del hematoma $7,8,10,13,17,18$.

\section{Bibliografía}

1. Brunori, A., Bruni, P., Mascioli, G., Chiapetta, F.: Severe lumbar stenosis caused by chronic spinal epidural hematoma and multiple disk disease - case report and review of the literature : Zentralbl Neurochir. 1994; 55: 166-171.

2. Gustafsson, H., Rutberg, H., Bengtsson, M.: Spinal Hematoma Following Epidural Analgesia: Report of a Patient with Ankylosing Spondylitis and a Bleeding Diathesis. Anaesthesia 1988; 43: 220-222.

3. Harik, S.I., Raichle, M.E., Reis, D.J.: Spontaneous Remitting Spinal Epidural Hematoma in a Patient on Anticoagulants. N Engl J Med 1971; 284: 1355-1357.

4. Imamura, T., Tsuburaya, K.: Chronic cervical epidural hematoma diagnosed by magnetic resonance imaging: No To Shinkei. 1990; 42: 857-861.

5. Kingery, W.S., Seibel, M., Date, E.S., Marks, M.P.: The natural resolution of a lumbar spontaneous epidural hematoma and associated radiculopathy. Spine. 1994; 19: 67-69.

6. Kotilainen, E.M., Pajulo, O.: Spontaneous epidural hematoma as a cause of sciatic pain in a schoolboy. Pediatr Neurol. 1997 ; 17: 350-352.

7. Lunardi, P., Mastronardi, L., Lo Bianco, F., Schettini, G., Puzzilli, F.: Chronic spontaneous spinal epidural hematoma simulating lumbar stenosis.: Eur Spine J. 1995; 4: 6466.

8. Mirkovic, S., Melany, M.: A thoracolumbar epidural hematoma simulating a disc syndrome.: J Spinal Disord. 199; 5: 112-115.

\section{Comentario al trabajo Hematoma epidural lumbar cró- nico espontáneo de J.M. Belinchón y cols.}

El interés de este caso radica, a mi entender, en que afecta al diagnóstico diferencial de unos cuadros tan prevalentes en nuestra especialidad como son la compresión radicular y la compresión medular, si bien por su rareza
9. Nagata, K., Ariyoshi, M., Ishibashi, K., Hashimoto, S., Inoue, A.: Chronic lumbar epidural hematoma in a patient who had spondylolysis at the third lumbar vertebra. Report of a rare case involving a seventeen-year-old adolescent: J Bone Joint Surg Am. 1998; 80: 1515-1520.

10. Nakagami, W., Yokota, S., Ohishi, Y., Ueda, H., Takahashi, Y., Sakuma, M., Ohshima, T., Kurita, K.: Chronic spontaneous lumbar spinal epidural hematoma. Spine. 1992; 17: 1509-1511.

11. Neurologic Complications of Regional Anesthesia. In Anesthesia for Obstetrics, Shnider S M and Levinson G, (eds.). Williams and Wilkins, Baltimore, $2^{\text {nd }}$ ed., 1987: pp 319320.

12. Packer, N.P., Cummins, B.H.: Spontaneous Epidural Hemorrhage: A Surgical Emergency. Lancet, 1978; 1: 356358.

13. Riffaud, L., Morandi, X., Chabert, E., Brassier, G.: Spontaneous chronic spinal epidural hematoma of the lumbar spine: J Neuroradiol. 1999; 26: 64-67.

14. Sage, D.J.: Epidurals, Spinals and Bleeding Disorders in Pregnancy: A Review. Anaesth Intens Care 1990; 18: 319-326.

15. Silber, S.H.: Complete Nonsurgical Resolution of a Spontaneous Spinal Epidural Hematoma. Am J Emergency Med 1996; 14: 391-393.

16. Tekkok, I.H., Cataltepe, K., Tahta, K., et al.: Extradural Hematoma After Continuous Extradural Anesthesia. Brit J Anaesth 1991; 67: 112-115.

17. Vázquez-Barquero, A., Abascal, F., García-Valtuille, R., Pinto, J.I., Figol, F.J., Cerezal, L.: Chronic nontraumatic spinal epidural hematoma of the lumbar spine: MRI Diagnosis. Eur Radiol. 2000; 10: 1602-1605.

18. Watanabe, N., Ogura, T., Kimori, K., Hase, H., Hirasawa, Y.: Epidural hematoma of the lumbar spine, simulating extruded lumbar disk herniation: clinical, discographic, and enhanced magnetic resonance imaging features. A case report: Spine. 1997; 22: 105-109.

Belinchón, J.M.; Campos, J.; Merino, J.; Gallego, J.M.; Barcia, C.: Hematoma epidural lumbar crónico espontáneo Neurocirugía 2005; 16: 533-536.

Correspondencia postal: J.M. Belinchón. Servicio de Neurocirugía. Consorcio Hospital General Universitario, Universidad de Valencia. Av. Tres Cruces s/n. 46014 Valencia, Spain.

habría que incluirlo en el grupo de miscelánea.

Tan sólo son 14 los casos descritos en la literatura de hematoma epidural crónico raquídeo según el reciente artículo de Stendel y col ${ }^{1}$; si bien 7 de ellos tienen antecedente 
traumático.

La causa del hematoma epidural espontáneo (es decir, aquel que no está relacionado con traumatismo, tumores, punción lumbar, anestesia raquídea o cirugía aunque algunos autores también excluyen los relacionados con trastornos de la coagulación o malformaciones vasculares) $)^{2,3}$, podría ser un aumento súbito de la presión abdominal que se transmite hacia las venas epidurales por su especial anatomía, que se podrían romper si estuviesen debilitadas ${ }^{1}$.

Por otra parte, que los hematomas epidurales crónicos raquídeos sean más frecuentes, en realidad exclusivos, de la región lumbar no es casual ya que la cronificación es posible por la tolerancia de la cauda equina comparada con la médula espinal y por la amplitud del canal a este nivel ${ }^{1}$.

\section{Bibliografía}

1. Stendel, R., Danne, M., Schulte, T., Stoltenburg-Didinger, G., Brock, M.: Chronic lumbar epidural haematoma presenting with acute paraparesis. Acta Neurochir 2003;145: 1015-1018.

2. Kingery, W.S., Seibel, M., Date, E.S., Marks, M.P.: The natural resolution of a lumbar spontaneous epidural hematoma and associated radiculopathy. Spine 1994;19: 67-69.

3. Clarke, D.B., Bertrand, G., Tampieri, D.: Spontaneous spinal epidural hematoma causing paraplegia: resolution and recovery without surgical decompression. Neurosurgery 1992; 30: 108-111. 